Edinburgh Preprint: 95/559

Liverpool Preprint: LTH-363

hep-lat/9511031

\title{
The Landshoff-Nachtmann Pomeron on the Lattice
}

\author{
UKQCD Collaboration \\ D.S. Henty, C. Parrinello] and D.G. Richards \\ Department of Physics and Astronomy, University of Edinburgh, Edinburgh \\ EH9 3JZ, Scotland
}

\begin{abstract}
We investigate the Landshoff-Nachtmann two-gluon-exchange model of the Pomeron using gluon propagators computed in the Landau gauge within quenched lattice QCD calculations. We first determine an effective gluon-quark coupling by constraining the Pomeron-quark coupling to its phenomenological value $\beta_{0}=2 \mathrm{GeV}^{-1}$. We then provide predictions for a variety of diffractive processes. As the propagators have been evaluated entirely from QCD first principles (although in the quenched approximation), our results provide a consistency check of the Landshoff-Nachtmann model. We address the issue of the possible gauge-dependence of our results, which will be the object of a future study.
\end{abstract}

\footnotetext{
${ }^{1}$ Present address: D.A.M.T.P., University of Liverpool, Liverpool L69 3BX, U.K.
} 


\section{Introduction}

The description of diffractive hadronic physics in terms of the exchange of a "Pomeron" has proved remarkably durable, and has successfully withstood the advent of QCD. Interest in the Pomeron has been renewed by recent results from HERA.

Total cross sections and diffractive processes are essentially soft in nature, and thus do not fall within the realm of perturbative QCD. Thus any fixed-order perturbative calculation must be regarded purely as a model of the interaction, but one which may be representative of an all-orders QCD result. Nevertheless, a successful phenomenological model of Pomeron exchange is provided by twogluon-exchange (2GE) [1, 2]. Crucial to this model is that the gluons be nonperturbative, i.e. infra-red finite, both to avoid the Coulomb singularity at $t=0$, and to provide the correct $t$-dependence of the differential cross section [3, 4.

Recently there has been a series of papers [5, 6] investigating the phenomenology of the 2GE model in the form proposed by Landshoff and Nachtmann (LN) [4]. In these papers a non-perturbative gluon propagator extracted from the solution of the Schwinger-Dyson equation [7] is inserted in the LN model. Here we will explore the phenomenology of the same model, using gluon propagators extracted from lattice calculations in the Landau gauge.

Our method has the advantage that one is inserting in the model a genuine, nonperturbative QCD quantity, which has been computed from first principles rather than derived from an approximate equation. Also, as we will explain in the following, we have only one free parameter in our approach, whereas the Schwinger-Dyson solution depends on two parameters. For these reasons, we think that our approach provides in principle the best chance to test the success of the LN model from the point of view of QCD.

The layout of the remainder of this paper is as follows. In the next section we will summarise the lattice calculations, and present the results for the lattice gluon propagator. In section 3 we will describe how we apply the measured gluon propagator to the calculation of cross-sections, and compare our results to the experimental data. In section 4 we comment on the issue of gauge dependence of our results. Finally we present our conclusions.

\section{Computational details}

We use the standard lattice definition of the gluon fields in terms of the link variables [8],

$$
A_{\mu}(x)=\frac{U_{\mu}(x)-U_{\mu}^{\dagger}(x)}{2 i a g_{0}}-\frac{1}{3} \operatorname{Tr}\left(\frac{U_{\mu}(x)-U_{\mu}^{\dagger}(x)}{2 i a g_{0}}\right),
$$




\begin{tabular}{|c|c|c|c|c|}
\hline & $\beta$ & Size & No. Cfgs. & $a^{-1}(\mathrm{GeV})$ \\
\hline This work & 6.0 & $16^{4}$ & 150 & 1.9 \\
\hline This work & 6.2 & $16^{4}$ & 150 & 2.7 \\
\hline Reference 【10 & 6.0 & $24^{3} \times 48$ & 500 & 1.9 \\
\hline
\end{tabular}

Table 1: Parameters of the lattices used in the calculation. The quoted value of the lattice spacing is obtained from an analysis of the string tension.

where $a$ is the lattice spacing and $g_{0}$ the bare coupling constant. The gauge configurations are fixed to the Landau gauge by imposing the gauge-fixing condition

$$
\Delta(x)=\sum_{\mu} A_{\mu}\left(x+\hat{e}_{\mu}\right)-A_{\mu}(x)=0 .
$$

The accurate implementation of this step is crucial, and we use the Fourieraccelerated algorithm of reference [9].

The unrenormalised gluon propagator in momentum space is obtained from the gauge-fixed gluon fields by taking the Fourier transform

$$
D_{\mu \nu}(p)=\left\langle A_{\mu}(p) A_{\nu}(-p)\right\rangle \text {. }
$$

In the continuum limit, we can write the Landau-gauge propagator in the form

$$
D_{\mu \nu}(p)=\left\{g_{\mu \nu}-\frac{p_{\mu} p_{\nu}}{p^{2}}\right\} D_{\text {lat }}(p) .
$$

We performed calculations on two sets of hypercubic lattices, corresponding to different physical volumes and lattice spacings. In addition, we used data for the gluon propagator as evaluated in Reference [10]; such data are particularly useful for an analysis of finite volume effects in our calculations. The parameters used are listed in Table 1. Full details of the method and of the simulation for the hypercubic lattices are contained in ref. [11].

As is customary in lattice QCD, we fix the value of the lattice spacing in units of energy from string tension measurements. This sets the scale of momenta for the gluon propagator.

A representative propagator, $D_{\text {lat }}(p)$, is shown in Figure (1). It is important to keep in mind that by defining the theory on a finite lattice we introduce both an ultraviolet and an infrared cutoff for Greens functions. For this reason, our gluon propagator is infrared finite by construction. On the other hand, when the lattice momenta are such that $|p a| \geq 1$, the theory becomes sensitive to the ultraviolet cutoff and one has to worry about finite lattice spacing effects. It is therefore crucial to probe the sensitivity of our results to the values of the cutoff scales by changing the lattice parameters as discussed above. 


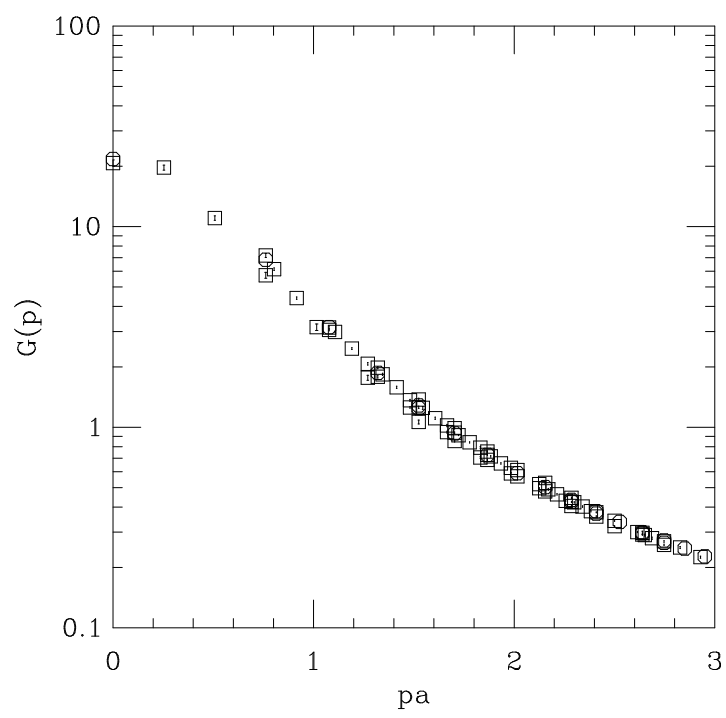

Figure 1: The gluon propagator on a $16^{4}$ lattice at $\beta=6.0$.

\begin{tabular}{|c|c||c|c|c||c|c|}
\hline$\beta$ & Size & $a_{0}$ & $a_{1}$ & $a_{2}$ & $b_{0}$ & $b_{1}$ \\
\hline 6.0 & $16^{4}$ & $3.37(1)$ & $0.176(1)$ & $0.445(5)$ & $3.139(4)$ & $0.193(1)$ \\
\hline 6.2 & $16^{4}$ & $2.72(1)$ & $0.122(1)$ & $0.461(5)$ & $2.645(4)$ & $0.133(1)$ \\
\hline
\end{tabular}

Table 2: Parameters of the fit to the gluon propagator on the hypercubic lattices, in units of the lattice spacing, as discussed in the text

We fit the propagator to the form

$$
D_{\text {lat }}(p)=\left\{\begin{array}{ll}
a_{0} /\left(a_{1}^{2}+p^{21+a_{2}}\right) & |p a| \leq 1 \\
b_{0} /\left(p^{2^{1+b_{2}}}\right) & |p a| \geq 1
\end{array} .\right.
$$

The parameters of the fits in lattice units for each of our hypercubic lattices are shown in Table 2; for the remaining lattice, we use the parameters quoted in Reference [10].

\section{Results}




\subsection{Determination of an effective quark-gluon coupling}

The salient feature of the LN model is that Pomeron exchange between quarks behaves like a $C=+1$ photon-exchange diagram, with amplitude

$$
i \beta_{0}^{2}\left(\bar{u} \gamma_{\mu} u\right)\left(\bar{u} \gamma^{\mu} u\right)
$$

$\beta_{0}$ represents the strength of the Pomeron coupling to quarks, and is related to the (non-perturbative) gluon propagator by

$$
\beta_{0}^{2}=\frac{1}{36 \pi^{2}} \int d^{2} p\left[g^{2} D(p)\right]^{2}
$$

where $g$ is the gluon-quark coupling. $\beta_{0}$ can be determined from, for example, the total $p p$ cross section 12, 13.

As a consequence, the LN model yields simple formulae for $p p$ scattering, exclusive $\rho$ production in deep inelastic scattering and the $J / \Psi$ - nucleon total cross

section, which all contain integrals in momentum space of $g^{2} D(p)$ [5, 6]. In order to extract predictions from the model, one needs an expression for $g^{2} D(p)$ which is valid both in the low momentum region $p \approx 1 \mathrm{GeV}$, where in fact the dominant contribution to the pomeron is expected, and for higher momenta, where the perturbative behaviour is recovered. Obviously, convergence of Equation (7) requires that the infra-red pole of the gluon propagator be removed by some nonperturbative mechanism.

In order to use the lattice gluon propagator $D_{\text {lat }}(p)$ in the LN model we make the following assumptions:

1. we neglect the running of the QCD coupling, i.e. we make the approximation $g(p)=g$;

2. we assume that in the continuum limit $D_{\text {lat }}(p)$ is multiplicatively renormalisable, as it is in perturbation theory.

As the scale for the momenta in $D_{\text {lat }}(p)$ is set from string tension measurements, our assumptions imply that we only have one free parameter to fix in the expression $g^{2} D_{\text {lat }}(p)$. This is a multiplicative factor, that corresponds to the product of a gluon wavefunction renormalisation constant times a numerical value for $g^{2}$. We will call this parameter $g_{\text {eff }}^{2}$, although strictly speaking it is more than just a squared coupling constant.

$g_{\text {eff }}^{2}$ can be determined by using Equation (7) as a normalisation condition, i.e. by imposing that $\beta_{0}$ attains its phenomenological value of $2.0 \mathrm{GeV}^{-1}$ :

$$
\beta_{0}^{2}=\frac{1}{36 \pi^{2}} \int d^{2} p\left[g_{\text {eff }}^{2} D_{\text {lat }}(p)\right]^{2}=4 \mathrm{GeV}^{-2}
$$

It is the combination $g_{\text {eff }}^{2} D_{\text {lat }}(p)$ that we insert in the formulae of the LN model. 


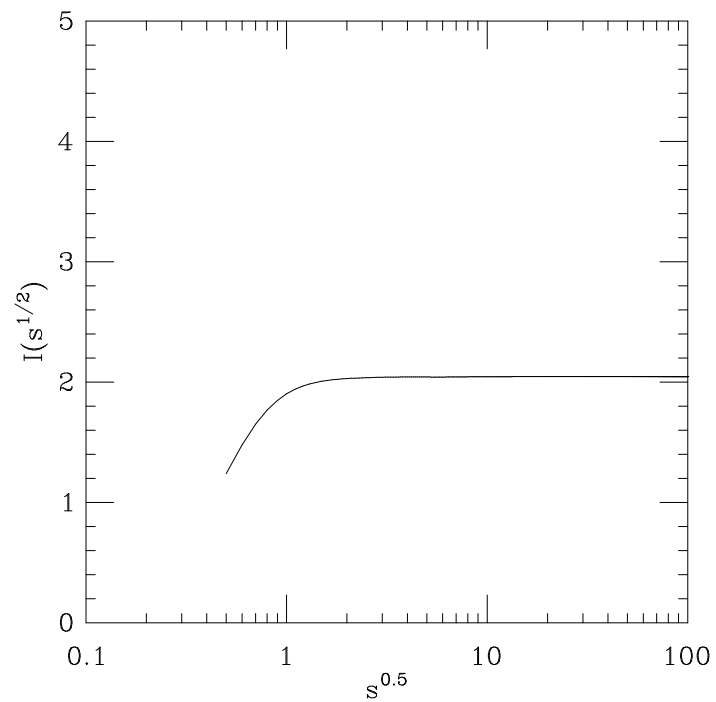

Figure 2: The integral $I\left(s^{1 / 2}\right)$ against $s^{1 / 2}$ on the $16^{4}$ lattice at $\beta=6.0$

As we mentioned in the previous section, one has to remember that the momentum dependence of the lattice gluon propagator is influenced by lattice artifacts. At high momentum, when $p$ becomes comparable to the ultraviolet cutoff $a^{-1}$, the propagator is affected by discretisation errors. In order to judge their likely importance in this calculation, we show in Figure (21) the integral

$$
I\left(s^{1 / 2}\right)=\frac{1}{36 \pi^{2}} \int_{0}^{s^{1 / 2}} d^{2} p\left[D_{\text {lat }}(p)\right]^{2},
$$

that we use to constrain $g_{\text {eff }}$, for the $16^{4}$ lattice at $\beta=6.0$. The dominant contribution to the integral indeed arises from momenta with $|p a|<1$, suggesting that our evaluation of the integral Equation (7) should suffer only weakly from discretisation uncertainties. A similar conclusion can be drawn from the other lattices in this study.

For low momenta, finite volume effects are present, making it difficult to identify a possible gluon mass. In order to isolate the extent to which such effects contaminate our calculation, we need a comparison with a calculation at a different lattice volume, but at the same lattice spacing. Such a comparison is provided by Reference [10], where a high-statistics measurement of the gluon propagator is made on a $24^{3} \times 32$ lattice at $\beta=6.0$. In the remainder of this paper, we will quote results using this determination of the lattice propagator, as well as our own determination. 


\begin{tabular}{|c|c|c|c|}
\hline & $\beta$ & Size & $\mu_{0}^{2}\left(\mathrm{GeV}^{2}\right)$ \\
\hline This work & 6.0 & $16^{4}$ & 0.85 \\
\hline This work & 6.2 & $16^{4}$ & 1.03 \\
\hline Reference [10] & 6.0 & $24^{3} \times 32$ & 0.93 \\
\hline
\end{tabular}

Table 3: The value of $\mu_{0}^{2}$ defined by Equation (10) on each lattice.

The inverse "radius" of the Pomeron, $\mu_{0}$, can be determined from the study of exclusive $\rho$-production in Deep Inelastic Scattering. It is related to the nonperturbative gluon propagator by

$$
\mu_{0}^{2}=\frac{\int d^{2} p p^{2}\left[g^{2} D(p)\right]^{2}}{\int d^{2} p\left[g^{2} D(p)\right]^{2}},
$$

and thus is independent of the coupling $g$ in our approximation, and furthermore should not depend on how we choose to parameterise the measured lattice gluon propagator. The phenomenological value is $\mu_{0}=1.1 \mathrm{GeV}$. The determination of $\mu_{0}$ on each lattice is shown in Table (3); the consistency between the different measurements is encouraging, and the results close to the phenomenological value.

We now have the ingredients to explore the phenomenology of the 2GE model: the $p^{2}$ dependence of the propagator determined from a lattice calculation, and an effective gluon-quark coupling determined from the fit to $\beta_{0}$. We begin the exploration with the analysis of elastic proton-proton scattering.

\subsection{Proton-proton elastic scattering}

The total and elastic differential cross section for proton-proton scattering provides a benchmark for the $2 \mathrm{GE}$ model of the Pomeron. Indeed it has already been explored extensively using nonperturbative propagators obtained from the approximate solution of the Schwinger-Dyson equation [15, 5]. We review the formalism here, and then apply the model using the lattice gluon propagator.

The 2GE amplitude for elastic proton-proton scattering can be written 16, 17]

$$
A(s, t)=i s 8 \alpha_{s}^{2}\left[T_{1}-T_{2}\right]
$$

with

$$
\begin{aligned}
T_{1}= & \int_{0}^{s} d^{2} k D(q / 2+k) D(q / 2-k)\left[G_{p}(q, 0)\right]^{2} \\
T_{2}= & \int_{0}^{s} d^{2} k D(q / 2+k) D(q / 2-k) G_{p}(q, k-q / 2) \\
& {\left[2 G_{p}(q, 0)-G_{p}(q, k-q / 2)\right] }
\end{aligned}
$$




\begin{tabular}{|c||c|c|c|c|}
\hline & $\beta$ & Size & $\sigma_{\text {tot }}^{0}(\mathrm{mb})$ & $B\left(\mathrm{GeV}^{-2}\right)$ \\
\hline This work & 6.0 & $16^{4}$ & 18.12 & 13.6 \\
\hline This work & 6.2 & $16^{4}$ & 19.21 & 12.9 \\
\hline Reference 【10 & 6.0 & $24^{3} \times 32$ & 19.85 & 12.6 \\
\hline
\end{tabular}

Table 4: The energy-independent total cross section, $\sigma_{\text {tot }}^{0}$, and the logarithmic slope parameter, $B$.

where $G_{p}(q, k)$ represents a convolution of proton wave functions. Here $T_{1}$ and $T_{2}$ represent the contributions when both gluons attach to the same quark and to different quarks within the proton respectively; our expectation [4, motivated by the additive quark rule, is that $T_{1}$ should dominate $T_{2}$. Following ref. [15], we take $G_{p}(q, k-q / 2)=F_{1}\left(q^{2}+\left|k^{2}-q^{2} / 4\right|\right)$, where $F_{1}$ is the elastic isoscalar form factor of the proton,

$$
F_{1}(t)=\frac{4 m^{2}-2.79 t}{\left(4 m^{2}-t\right)(1-t / 0.71)^{2}}
$$

The total and elastic differential cross-sections are given by

$$
\begin{aligned}
\sigma_{\text {tot }}^{0} & =\frac{A(0)}{i s} \\
\frac{d \sigma^{0}}{d t} & =\frac{|A(t)|^{2}}{16 \pi s^{2}}
\end{aligned}
$$

respectively. Single Pomeron exchange is expected to dominate the differential cross-section out to $-t \simeq 0.5 \mathrm{GeV}^{2}$. To fully describe the energy dependence, the intercept of the Pomeron trajectory is taken to be somewhat larger than 1 , and the measured total and differential cross sections are related to the energyindependent expressions above by

$$
\begin{aligned}
\sigma_{\mathrm{tot}} & =\left(\frac{s}{m_{p}^{2}}\right)^{0.08} \sigma_{\mathrm{tot}}^{0} \\
\frac{d \sigma}{d t} & =\left(\frac{s}{m_{p}^{2}}\right)^{0.168} \frac{d \sigma^{0}}{d t}
\end{aligned}
$$

For small $t$, the elastic differential cross section behaves like $e^{B t}$, and the model is characterised by two parameters, $\sigma_{\text {tot }}^{0}$ and $B$.

Following the discussion in the previous subsection, we compute $\sigma_{\text {tot }}^{0}$ and $B$ on each lattice using the lattice gluon propagator and the effective coupling $g_{\text {eff }}$. Our results are presented in Table 4. The general agreement between the values of $\sigma_{\text {tot }}^{0}$ and $B$ obtained on the various lattices is striking, suggesting either that 


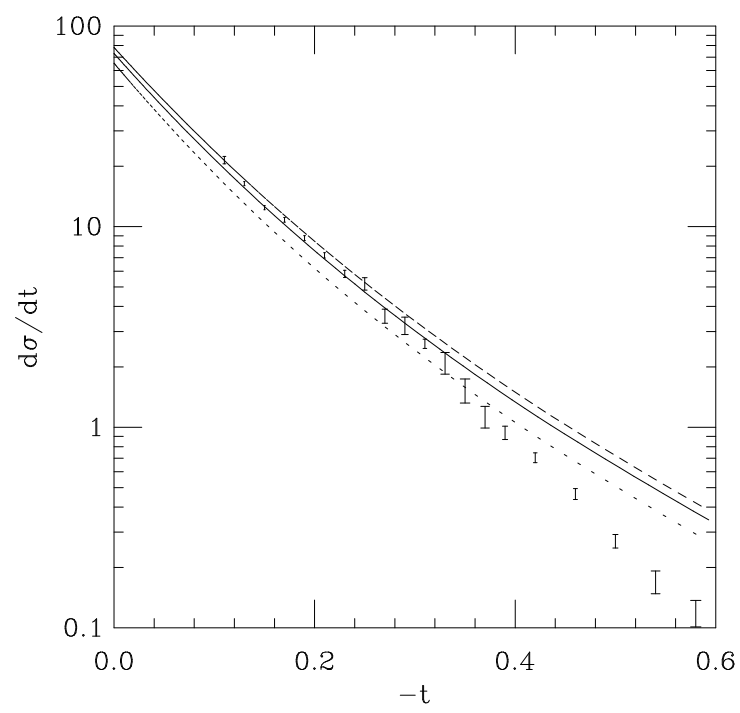

Figure 3: Data for the $p p$ elastic cross section at $\sqrt{s}=53 \mathrm{GeV}$ from Ref. [18], together with the lattice prediction corrected for energy dependence on the $16^{4}$ lattices at $\beta=6.2$ (solid) and $\beta=6.0$ (dots), and on the $24^{3} \times 32$ lattice at $\beta=6.0$ (dashes).

both quantities are subject to only small discretisation or finite volume effects, or that such effects are absorbed in $g_{\text {eff }}$. They are also encouragingly close to the phenomenological values of $\sigma_{\text {tot }}^{0} \simeq 22.7 \mathrm{mb}$ and $B \sim 11 \mathrm{GeV}^{-2}$.

The quality of the fit to the data can be seen in Figure (3), where we show ISR data for the differential elastic cross section at $\sqrt{s}=53 \mathrm{GeV}$ together with the lattice predictions, with the energy correction of Equation (18). Though the agreement of the lattice computation with the experimental data is good, differences between the lattice results are now clearer, and suggest the need to repeat the calculation at different volumes and at different lattice spacings. In particular, our lattice at $\beta=6.2$ corresponds to a somewhat tiny physical volume for hadronic physics, so that finite volume effects may be non-negligible in that case.

\subsection{J/ $/$-Nucleon Scattering}

This process provides a further important test of the $2 \mathrm{GE}$ model, and in the following we adopt the analysis procedure of Ducati et al. [6]. The amplitude for 


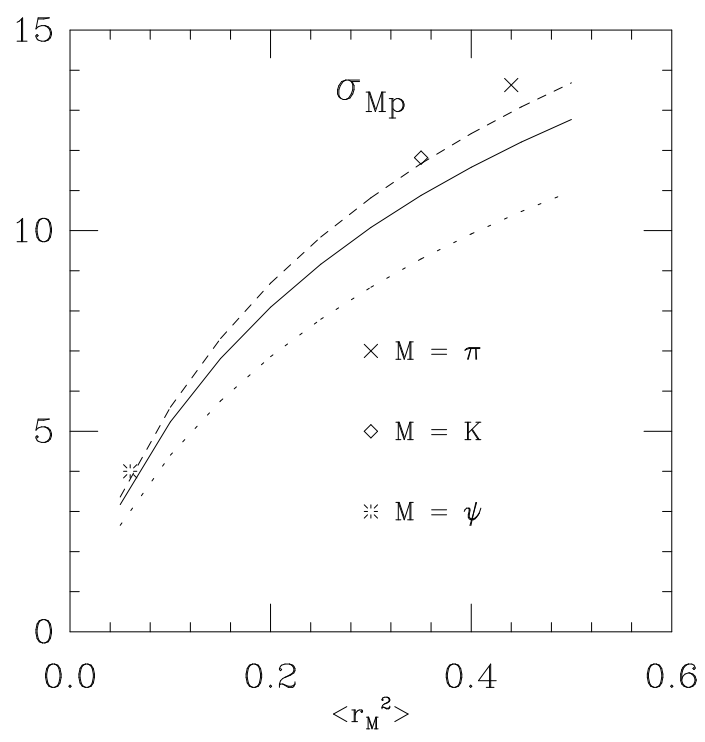

Figure 4: The meson-nucleon total cross section as a function of the radius used in the meson form factor, Equation (20), on the $16^{4}$ lattices at $\beta=6.2$ (solid) and $\beta=6.0$ (dots), and on the $24^{3} \times 32$ lattice at $\beta=6.0$ (dashes). Also shown are the radii and total cross sections corresponding to the $\pi, K$ and $J / \psi$.

meson-nucleon scattering is

$$
\begin{aligned}
& A(s, t)=i s \frac{32}{9} \alpha_{s}^{2} \int_{0}^{s} d^{2} k D(k) D(2 q-k) \\
& \quad 2\left[f_{M}\left(q^{2}\right)-f_{M}\left((q-k)^{2}\right)\right] 3\left[f_{N}\left(q^{2}\right)-f_{N}\left(q^{2}-\frac{3}{2} \vec{q} \cdot \vec{k}+\frac{3}{4} k^{2}\right)\right] .
\end{aligned}
$$

We use the pole approximation for the form factors

$$
f_{i}\left(k^{2}\right)=\frac{1}{\left(1-\frac{1}{6} k^{2}\left\langle r_{i}^{2}\right\rangle\right)},
$$

taking [19] $\left\langle r_{p}^{2}\right\rangle=0.67 \mathrm{fm}^{2},\left\langle r_{\pi}^{2}\right\rangle=0.44 \mathrm{fm}^{2},\left\langle r_{K}^{2}\right\rangle=0.35 \mathrm{fm}^{2}$ and $\left\langle r_{J / \psi}^{2}\right\rangle=$ $0.04 \mathrm{fm}^{2}$.

Two phenomenological features are evident in the calculation. Firstly, the quark-counting rule is closely satisfied in the case of the hadrons composed of light quarks:

$$
\begin{array}{rlrl}
\beta & =6.0, & 16^{4}: & \sigma_{p p} / \sigma_{\pi p}=1.8, \\
\beta=6.2, & 16^{4}: & \sigma_{p p} / \sigma_{\pi p}=1.6, \\
\beta=6.0, & 24^{3} \times 32: & \sigma_{p p} / \sigma_{\pi p}=1.5 .
\end{array}
$$


Secondly, the Pomeron couples more weakly to mesons composed of heavier quarks. This can be seen in Figure (团) where we show the meson-nucleon cross section as a function of the pole radius used in Equation (20), together with a Regge fit to the energy-independent part of the $\pi^{-} p$ and $K^{-} p$ cross sections performed in Reference [20]. The differences between the results on the different lattices reinforces the need to repeat the calculation on a wider range of lattice parameters.

\section{Gauge Dependence}

The issue of gauge dependence in the LN model is a difficult one. Indeed, the starting point of LN is the leading-order perturbative calculation of a quark-quark scattering amplitude, with the constraint that no colour is exchanged between quarks. This is of course a gauge invariant quantity, expressed by a two-gluon exchange diagram. On the other hand, LN argue that the salient features of pomeron physics can be captured by substituting in such a diagram the tree-level gluon propagator with an effective one, including non-perturbative self-energy effects, which should in particular account for the removal of the infrared pole. Clearly any ansatz for the self-energy introduces in principle an uncontrollable gauge dependence in the calculation. Given the phenomenological success of the model, one is led to think that maybe the gauge dependence intrinsic in the propagator is either mild by itself or gets suppressed (cancels) in the evaluation of physical quantities. We emphasise that this problem is inherent in the model and has nothing to do with using a lattice propagator. From the point of view of our method, one may speculate that the simplest conceivable mechanism for the suppression of gauge dependence would be if it gets "factored out" in the definition of $g_{\text {eff }}$. In other words, a gauge dependent definition of $g_{\text {eff }}$ may cancel most of the gauge dependence in the momentum space integrals of the propagator.

We plan to investigate directly the issue of gauge dependence in a future work by using lattice propagators obtained in different gauges.

\section{Conclusions}

We have demonstrated how the Landau-gauge lattice gluon propagator employed in the two-gluon-exchange model of proton-proton scattering provides a highly successful description of the data, where the only parameter that needs to be fixed is an effective quark-gluon coupling. For the first time a genuine QCD quantity, evaluated entirely from first principles, has been inserted in the LN model, providing an important consistency check of the model itself. Also, given the fact that in our approximation the effect of quark loops diagrams is absent, our analysis shows the robustness of the LN model, despite its simplicity. 
Although the description of the data appears largely independent both of the volume of the lattice and of the lattice spacing, a more detailed analysis of lattice systematic errors is necessary. We plan to perform such an analysis, together with an investigation of the gauge dependence of our results, in a future publication.

\section{Acknowledgements}

We wish to thank Sandy Donnachie and Peter Landshoff for useful conversations, and Chris Michael both for helpful conversations and for reading the manuscript. This work was supported by the United Kingdom Particle Physics and Astronomy Research Council (PPARC) under grant GR/J21347. CP and DGR acknowledge the support of PPARC through Advanced Fellowships held at the University of Liverpool and at the University of Edinburgh respectively. We are grateful to Edinburgh's Computing Service for maintenance of service on the Thinking Machines CM-200 where this work was performed.

\section{References}

[1] F.E. Low, Phys. Rev. D12 (1975) 163.

[2] S. Nussinov, Phys. Rev. Lett. 34 (1975) 1268.

[3] D.G. Richards, Nucl. Phys. B258 (1985) 267.

[4] P.V. Landshoff and O. Nachtmann, Z. Phys. C35 (1987) 405.

[5] F. Halzen, G. Krein and A.A. Natale, Phys. Rev. D47 (1993) 295.

[6] M.B. Gay Ducati, F. Halzen and A.A. Natale, Phys. Rev. D48 (1993) 2324.

[7] J.M. Cornwall, Phys. Rev. D26 (1982) 1453.

[8] J. Mandula and M. Ogilvie, Phys. Lett. B185 (1987)

[9] C.T.H. Davies et al., Phys. Rev. D37 (1987) 1581.

[10] P. Marenzoni, G. Martinelli, and N. Stella, Rome Preprint 94/1042, Southampton Preprint 93/94-31, hep-lat/9410011.

[11] D.S. Henty et al., Liverpool Preprint LTH-361, Orsay Preprint LPTHE 9567, hep-lat/9510045, to appear in the Proceedings of the International Europhysics Conference on High Energy Physics (HEP 95), Brussels, Belgium, 27th. Jul. - 2nd. Aug., 1995

[12] A. Donnachie and P.V. Landshoff, Nucl. Phys. B244 (1984) 322. 
[13] A. Donnachie and P.V. Landshoff, Nucl. Phys. B267 (1985) 690.

[14] A. Donnachie and P.V. Landshoff, Phys. Lett. B185 (1987) 403.

[15] J.R. Cudell and D.A. Ross, Nucl. Phys. B359 (1991) 247.

[16] J.F. Gunion and D. Soper, Phys. Rev. D15 (1977) 2617.

[17] E.M. Levin and M.G. Ryskin, Sov. J. Nucl. Phys. 34 (1981) 619.

[18] A. Breakstone et al., Nucl. Phys. B248 (1984) 253.

[19] B. Povh and J. Hufner, Phys. Rev. Lett. 58 (1987) 1612; Phys. Lett. B245 (1990) 653.

[20] A. Donnachie and P.V. Landshoff, Phys. Lett. B296 (1992) 227. 